\title{
Classifier combination approach for question classification for Bengali question answering system
}

\author{
SOMNATH BANERJEE ${ }^{1, *}{ }^{\mathbb{D}}$, SUDIP KUMAR NASKAR ${ }^{1}$, PAOLO ROSSO $^{2}$ and \\ SIVAJI BNDYOPADHYAY ${ }^{1}$ \\ ${ }^{1}$ Department of Computer Science and Engineering, Jadavpur University, Kolkata, India \\ ${ }^{2}$ PRHLT Research Center, Universitat Politècnica de València, Valencia, Spain \\ e-mail: sb.cse.ju@gmail.com
}

MS received 17 November 2017; revised 25 August 2019; accepted 9 September 2019

\begin{abstract}
Question classification (QC) is a prime constituent of an automated question answering system. The work presented here demonstrates that a combination of multiple models achieves better classification performance than those obtained with existing individual models for the QC task in Bengali. We have exploited stateof-the-art multiple model combination techniques, i.e., ensemble, stacking and voting, to increase QC accuracy. Lexical, syntactic and semantic features of Bengali questions are used for four well-known classifiers, namely Naïve Bayes, kernel Naïve Bayes, Rule Induction and Decision Tree, which serve as our base learners. Singlelayer question-class taxonomy with 8 coarse-grained classes is extended to two-layer taxonomy by adding 69 fine-grained classes. We carried out the experiments both on single-layer and two-layer taxonomies. Experimental results confirmed that classifier combination approaches outperform single-classifier classification approaches by $4.02 \%$ for coarse-grained question classes. Overall, the stacking approach produces the best results for fine-grained classification and achieves $87.79 \%$ of accuracy. The approach presented here could be used in other Indo-Aryan or Indic languages to develop a question answering system.
\end{abstract}

Keywords. Bengali question classification; question classification; classifier combinations.

\section{Introduction}

A question answering (QA) system is an automatic system capable of answering natural language questions in a human-like manner: with a concise, precise answer. With the explosion of information on the internet, research in QA is becoming increasingly important. QA is a research area that combines research from different, but related, fields like Information Retrieval (IR), Information Extraction (IE), Natural Language Processing (NLP), etc. QA is different from IR in the fact that the objective of QA is retrieving answers to questions while the goal of IR systems (i.e., search engines) is to just retrieve relevant documents. This implies that QA systems will possibly make the next generation search engines. According to [1], typically an automated QA system has three stages: question processing, passage retrieval and answer processing. In the question processing stage, the natural language question is analysed to create a proper IR query and also the entity type of the answer is detected. The first task is called query reformation and the second is called question classification (QC). In this work, we focus on QC, which is an important

*For correspondence

Published online: 03 December 2019 component of factoid QA systems. Factoid questions (e.g. Who founded Virgin Airlines?) are questions that can be answered with simple facts expressed in short text answers [2]. QC plays an important role in the QA framework, though different QA systems follow different architectures [3]. Furthermore, earlier studies [4-6] reported that QC has significant influence on the overall performance of the QA systems. The task of a QC module is to assign one or more class labels, depending on the classification strategy, to a given question written in a natural language. For example, for the question "Which London street is the home of British journalism?" the task of a QC component is to assign the label 'Location' to this question. Since it effectively predicts the type of the answer, QC is also often referred to as answer type prediction.

The two foremost motivations for QC are locating the answer and choosing the search strategy. Knowing the question class not only reduces the search space to be explored for finding the answer but it also helps find the true answer from a given set of candidate answers. On the other hand, the question class can also be used to choose the search strategy when the question is reformed to a query over IR engine. For example, consider the question "What is a pyrotechnic display?". Identifying that the question 
class is of type 'Definition', the searching template for locating the answer may be for example "pyrotechnic display is a ..." or "pyrotechnic displays are ...", which are much more effective than simply searching by the question words.

Although the QA systems developed for European languages, particularly in English, have achieved reasonable accuracy, the situation for the Indian languages is completely different. Research on QA has not been initiated for most of the Indian languages. Like other Indian languages, Bengali (also known as 'Bangla') presents serious challenges for QA. Bengali is an Indo-Aryan language such as Hindi, Marathi, Gujrati, etc. With about 193 million native and about 230 million total speakers, Bengali is one of the most spoken languages (ranked sixth) in the world and the second most commonly spoken language in India as per 2011 Census of India ${ }^{1}$. Due to the rapid increase of contents in Bengali on the web, the research communities have started to take notice and interest in Bengali. Unlike English, Bengali has many interrogatives [7]. Even in Bengali, the position of the interrogatives in the question text is not fixed due to relatively free phrase order of the language. Moreover, the language processing tools for Bengali are in the development phase.

One of the key issues of classification modelling is the enhancement of classification accuracy. In this regard, notable number of researchers have recently paid considerable attention to classifier combination methods. The idea is not to rely on a single decision making scheme. Instead, many single/individual classifiers are used for decision making by combining their individual opinions to arrive at a consensus decision.

The rest of the paper is structured as follows: we start with a discussion of the related work in section 2. We discuss the Bengali question taxonomies in section 3. The features for the classification task is described in section 4 . Section 5 discusses the detailed results. Finally, we conclude in section 6 .

\section{Related work}

A considerable volume of research has been carried out on $\mathrm{QC}$, question taxonomies and question features [8]. In the past decade, QC was enormously addressed. Broadly two different approaches are used to classify questions - rulebased $[9,10]$ and machine learning based $[11,12]$. However, a number of researchers have also employed a few hybrid approaches, which combine rule-based and machine-learning-based approaches [13, 14].

In rule-based approaches, manually handcrafted grammar rules are used to analyse a question in order to determine the answer type $[9,10]$. Although handcrafted rules

\footnotetext{
$\overline{{ }^{1} \mathrm{http} / / / w w w . c e n s u s i n d i a . g o v . i n / 2011 C e n s u s / L a n g u a g e-2011 / S t a t e ~}$ ment-1.pdf.
}

have been used successfully, however, designing these rules is expensive [15]. Li and Roth [15] stated that although rule-based approaches may perform well on a particular dataset but the classification performance may degrade on a new dataset and consequently it is difficult to scale them. Therefore, it is very much challenging to build a manual classifier with a limited number of rules. In contrast, machine-learning-based QC approaches are performed by extracting features from the questions, training a classifier and predicting the question class using the trained classifier. Many researchers employed machine learning techniques, e.g., maximum entropy [16], support vector machine [17], etc. using different features, such as syntactic features [12], semantic features [11], etc. However, these works were primarily focused on the factoid questions of English and restricted to classify the questions into two categories (namely, yes and no) or a few predefined categories (e.g., 'what', 'how', 'why', 'when', 'where' and so on).

Many researchers have investigated the technique of combining the predictions of multiple classifiers to build a single classifier [18-21]. It has been observed that the resulting classifier is generally more accurate than any of the individual classifiers making up the ensemble. Both theoretical [22, 23] and empirical [24-26] studies were carried out on classifier combination. A number of studies were carried out on classifier combination methods for the QC task in the last decade. Xin et al [27] trained four SVM classifiers based on four different types of features and combined them with various strategies. They compared Adaboost [28], Neural Networks and Transition-Based Learning (TBL) [29] combination methods on the trained classifiers. Their evaluation results on the TREC dataset revealed that the use of TBL combination method improved classification accuracy up to $1.6 \%$ compared with a single classifier trained on all features. Jia et al [30] proposed ensemble learning for Chinese QC. They translated and modified the UIUC (University of Illinois, Urbana Champaign) and TREC (Text REtrieval Conference) dataset to Chinese. The proposed method achieved $87.6 \%$ precision for fine-grained question types. The ensemble method has also been employed for Chinese QC [31]. The aforementioned experiments with bagging [18] and AdaBoost.M1 [28] algorithms showed that such approaches can effectively utilize multiple classifiers to improve the accuracy rate of QC than a single classifier.

Presently, QA systems developed for European [32-34], Middle Eastern [35-37] and Asian languages [38-41] are capable of providing answers with reasonable accuracy. However, the scenario is different for Indian languages, in which QA research is in a nascent stage. There are 22 official languages in India and for most of these Indian languages, research in QA has not been started yet. A factoid Hindi QA system named 'Prashnottar' was proposed in [42]. 'Prashnottar' uses handcrafted rules to identify question patterns for QC. Recently, [43] developed 
a QA system for Hindi, which uses Naïve Bayes (NB) technique for QC. They reported that the classifier was tested on 75 questions. A few Hindi-English cross-lingual QA systems also have been reported in the literature. In 2003, [44] developed a cross-lingual QA system for Hindi and English, which employed a rule-based approach for QC. A multilingual restricted domain QA system was reported in [45], which also uses a rule-based approach for classifying questions. [46] provides a review of the state-ofthe-art in Hindi QA, and it criticized that [45] did not report the test-set corpus statistics. In [47], a QA system in Hindi was reported. Based on the keywords, they applied rulebased approach for classifying the questions into six categories. For Telugu, [48] proposed a dialogue-based QA system for the railway domain. They used rule-based approach to classify questions. [49] reported a QA system developed for Punjabi. The work is based on a concept taken from physics: 'Point of Gravity'. However, they did not report any QC approach. [50] reports a QA system for Malayalam, which is based on named entity (NE) tagging and QC. A rule-based approach was adopted for QC. It can be concluded from the related work that a few QA systems have been developed for Indian languages and mainly rulebased approach has been employed for classifying questions.

Like other Indian languages, research in Bengali QA has received very less attention than its western and middleeastern counterparts. Although any QA system is not available in Bengali to date, a study [7] has been carried out on the Bengali QC task in which suitable lexical, syntactic and semantic features and Bengali interrogatives have been studied, a single-layer taxonomy of nine coarse-grained classes has been proposed and $87.63 \%$ QC accuracy has been reported. The proposed method used four classifiers independently, namely, Naïve Bayes (NB), kernel Naïve Bayes (k-NB), Rule Induction (RI) and Decision Tree (DT). Both theoretical [22, 23] and empirical [24-26] studies confirm that the classifier combination approach is generally more accurate than any of the individual classifiers making up the ensemble. Furthermore, a number of studies $[27,30]$ were successfully carried out on classifier combination methods for the QC task, which outperformed the individual classifiers. Therefore, we consider classifier combination for classifying Bengali questions. To the best of our knowledge, classifier combination methods have not been employed for the QC task for Indian languages, prior to the work reported in this paper. As discussed earlier, mainly rule-based approach was employed for the QC task along with an approach based on individual classifiers. Furthermore, no research work can be found in the literature for fine-grained QC in Bengali. The deep learning framework performs well when large datasets are available for training and the framework is less effective than traditional machine learning approaches when the training datasets are small in size. In this work, we deal with a dataset that has only 1,100 samples. Therefore, we prefer classifier combination approach over deep learning. Li and Roth [15] and Lee et al [51] proposed 50 and 62 finegrained classes for English and Chinese QC, respectively. In our work, we propose 69 fine-grained question classes to develop a two-layer taxonomy for Bengali QC.

\section{Proposed question taxonomies}

The set of question categories is referred to as question taxonomy or question ontology. Since Bengali QC is at an early stage of development, for simplicity initially a singlelayer taxonomy for Bengali question types has been proposed in [7], which consists of only eight coarse-grained classes and no fine-grained classes. No other investigations have been carried out for coarse-grained Bengali taxonomies to date. Later, based on the coarse-grained classes in [7], fine-grained question classes were proposed in [52]. Table 1 presents the Bengali question taxonomy proposed in $[7,52]$.

The taxonomy proposed by $\mathrm{Li}$ and Roth [15] contains 6 coarse-grained classes: Abbreviation, Description, Entity, Human, Location and Numeric. Abbreviation and Description classes of [15] are not present in Bengali taxonomy. Two coarse-grained classes of [15], namely Entity and Human have resemblance with Miscellaneous and Person, respectively, in Bengali taxonomy, while Location and Number classes are present in both the taxonomies, and Organization and Method classes are not present in [15]. In 2-layer Bengali taxonomy, 15 fine-grained classes of [15] are not present, namely abbreviation, expression, definition, description, manner, reason, event, letter, substance, title, description, state, code, distance and order.

All the coarse-grained classes of Lee et al [51] are present in Bengali taxonomy. However, the Method class of Bengali taxonomy is not present in [51]. The Artifact class of [51] is similar to Definition and Miscellaneous of Bengali taxonomy. In 2-layer Bengali taxonomy, 9 fine-grained classes of [51] are not included, namely, firstperson, planet, province, political system, substance, range, number, range and order.

Five fine-grained classes are introduced in Bengali taxonomy that are not present in [15] and [51]. The five classes are AGE, NATURAL, ARTIFICIAL, INSTRUMENTAL and NON-INSTRUMENTAL. The NATURAL and ARTIFICIAL fine-grained classes belong to Method coarse-grained class, which is not present in [15] and [51]. Similarly, INSTRUMENTAL and NON-INSTRUMENTAL fine-grained classes belong to the Reason coarse-grained class. Also, the Reason coarse-grained class is not present in [15] and [51]. The $A G E$ fine-grained class belongs to Numerical coarse class.

The taxonomies proposed in [15] and [51] did not deal with causal and procedural questions. The proposed Bengali 2-layer taxonomy is based on the only available Bengali QA dataset [7], which contains causal and procedural 
Table 1. Two-layer Bengali question taxonomies.

\begin{tabular}{|c|c|}
\hline Coarse-grained & Fine-grained \\
\hline Person (PER) & $\begin{array}{l}\text { GROUP, INDIVIDUAL, APPELLATION, INVENTOR/ DISCOVERER, POSITION, } \\
\text { OTHER }\end{array}$ \\
\hline Organization (ORG) & BANK, COMPANY, SPORT-TEAM, UNIVERSITY, OTHER \\
\hline Location (LOC) & $\begin{array}{c}\text { CITY, CONTINENT, COUNTRY, ISLAND, LAKE, MOUNTAIN, OCEAN, } \\
\text { ADDRESS, RIVER, OTHER }\end{array}$ \\
\hline Temporal (TEM) & DATE, TIME, YEAR, MONTH, WEEK, DAY, OTHER \\
\hline Numerical (NUM) & $\begin{array}{l}\text { AGE, AREA, COUNT, LENGTH, FREQUENCY, MONEY, PERCENT, PHONE- } \\
\text { NUMBER, SPEED, WEIGHT, TEMPERATURE, OTHER }\end{array}$ \\
\hline Method (METH) & NATURAL, ARTIFICIAL \\
\hline Reason (REA) & INSTRUMENTAL, NON-INSTRUMENTAL \\
\hline Definition (DEF) & $\begin{array}{l}\text { ANIMAL, BODY, CREATION, CURRENCY, FOOD, INSTRUMENT, OTHER, } \\
\text { PLANT, PRODUCT, SPORT, SYMBOL, TECHNIQUE, TERM, WORD }\end{array}$ \\
\hline Miscellaneous (MISC) & $\begin{array}{c}\text { COLOR, CURRENCY, ENTERTAINMENT, LANGUAGE, OTHER, VEHICLE, } \\
\text { AFFAIR, DISEASE, PRESS, RELIGION }\end{array}$ \\
\hline
\end{tabular}

questions. Therefore, the Bengali taxonomy contains question classes of causal and procedural questions. A few fine-grained classes of [15] and [51] are not included in the taxonomy because such questions are not present in the Bengali QA dataset. However, the proposed Bengali taxonomy is not final for Bengali QA task. Increasing the size of the said dataset is still in the process. Therefore, it is expected that the missing fine-grained classes will be incorporated in the taxonomy in future.

\section{Features for QC}

In the task of machine-learning-based QC, deciding the optimal set of features to train the classifiers is crucial. The features used for the QC task can be broadly categorized into three different types: lexical, syntactic and semantic features [53]. In the present work, we also employed these three types of features suitable for the Bengali QC task.

Loni et al [53] represented questions for the QC task similar to document representation in the vector space model, i.e., a question is represented as a vector described by the words inside it. Therefore, a question $Q_{i}$ can be represented as follows:

$$
Q_{i}=\left(W_{i 1}, W_{i 2}, W_{i 3}, \ldots, W_{i(N-1)}, W_{i N}\right)
$$

where $W_{i k}$ is frequency of the term $k$ in question $Q_{i}$ and $N$ is total number of terms.

Due to the sparseness of the feature vector, only non-zero valued features are kept. Therefore, the size of the samples is quite small despite the huge size of feature space. All lexical, syntactic and semantic features can be added to the feature space, which expands the feature vector.

In the present study, the features employed for classifying questions (see table 1) are described in the following subsections. In addition to the features used for the coarsegrained classification, fine-grained classification uses an additional feature, namely coarse class, i.e. label of the coarse-grained class.

\subsection{Lexical features}

Lexical features $\left(f_{L}\right)$ of a question are extracted from the words appearing in the question. Lexical features include interrogative-words, interrogative-word-positions, interrogative-type, question-length, end-marker and wordshape.

- Interrogative-words and interrogative-word positions: The interrogative-words (e.g., what, who, which, etc.) of a question are important lexical features. They are often referred to as wh-words. Huang et al [13, 54] showed that considering question interrogativeword(s) as a feature can improve the performance of QC task for English QA. Because of the relatively free word-ordering in Bengali, interrogative-words might not always appear at the beginning of the sentence, as in English. Therefore, the positions of the interrogative (wh) words along with the interrogative words themselves have been considered as the lexical features. The position value is based on the appearance of the interrogative word in the question text and it can have any of the three values namely, first, middle and last.

- Interrogative-type: Unlike in English, there are many interrogatives present in the Bengali language. Twenty six Bengali interrogatives were reported in [7]. In the present work, the Bengali interrogative-type (wh-type) is considered as another lexical feature. In [7], the authors concluded that Bengali interrogatives not only provide important information about the expected answers but also indicate the number information 
(i.e., singular vs plural). In [7], wh-type was classified to three categories: simple interrogative (SI) or unit interrogative (UI), dual interrogative (UI) and compound/composite interrogative (CI).

- Question length: Blunsom et al [55] introduced the length of a question as an important lexical feature which is simply the number of words in a question. We also considered this feature for the present study.

- End marker: The end marker plays an important role in Bengali QC task. Bengali question ends with either '?' or ' $l$ '. It has been observed from the experimental corpus that if the end marker is 'I' (similar to dot (.) in English), then the given question is a definition question.

- Word shape: The word shape of each question word is considered as a feature. Word shapes refer to apparent properties of single words. Huang et al [13] introduced five categories for word shapes: all digits, lower case, upper case, mixed and other. Word shape alone is not a good feature for QC; however, when it is combined with other kinds of features, it usually improves the accuracy of QC $[13,53]$. Capitalization feature is not present in Bengali; hence, we have considered only the other three categories, i.e., all digits, mixed and other.

\section{Example-1: ke gOdZa prawiRTA karena? ${ }^{2}$ \\ Gloss: Who established Goura?}

Lexical features: wh-word: ke; wh-word position: first; wh-type: SI; question length: 5; end marker:? word shape: other

\subsection{Syntactic features}

Although different works extracted several syntactic features $\left(f_{S}\right)$, the most commonly used $f_{S}$ are Part of Speech (POS) tags and head-words [8].

- POS tags: In the present work, we used the POS tag of each word in a question such as NN (noun), JJ (adjective), etc. POS of each question word is added to the feature vector. A similar approach was successfully used for English [15, 55]. This feature space is sometimes referred to as the bag-of-POS tags [53]. The tagged-unigram (TU) feature was formally introduced by [53]. TU feature is simply the unigrams augmented with POS tags. Loni et al [53] showed that considering the TGs instead of normal unigrams can help the classifier to distinguish a word with different tags as two different features. For extracting the POS tags, the proposed classification work in Bengali uses a

\footnotetext{
${ }^{2}$ All the Bengali examples in this paper are written in WX [56] notation which is a transliteration scheme for representing Indian languages in ASCII.
}

Bengali Shallow Parser, ${ }^{3}$ which produces POS tagged data as intermediate result.

- Question head-word: Question head-word is the most informative word in a question as it specifies the object the question is looking for [13]. Correctly identifying head-words can significantly improve the classification accuracy. For example, in the question "What is the oldest city in Canada?" the head-word is "city'. The word 'city' in this question can highly contribute to classify this question as LOC: city.

Identifying the question's head-word is very challenging in Bengali because of its syntactic nature and no research has been conducted so far on this. Based on the position of the interrogative in the question, we use heuristics to identify the question head-words. According to the position of the interrogative, three cases are possible.

- Position I (at the beginning): If the question-word (i.e., marked by WQ tag) appears at the beginning then the first NP chunk after the interrogative-word is considered as the head-word of the question. Let us consider the following question.

Example-2: $k e(/ \mathrm{WQ}) \operatorname{gOdZa(/\mathrm {NP})}$ prawiRTA(/ $\mathrm{NN})$ karena(/VM)?(/SYM)

English gloss: Who established Goura?

In th example, gOdZa is the head-word.

- Position II (in between): If the position of the question-word is neither at the beginning nor at the end, then the immediate NP-chunk before the interrogative-word is considered as the head-word. Let us consider the following question.

Example-3: $g O d Z a(/ \mathrm{NNP})$ koWAyZa(/WQ) aba$\operatorname{shiwa(/JJ)?(/SYM)}$

English gloss: Where is Goura situated?

In the above example gOdZa is considered as the question head-word.

- Position III (at the end): If the question-word appears at the end (i.e., just before the end of sentence marker) then the immediate NP-chunk before the interrogative-word is considered as the question head-word. Therefore, a similar action is taken for Positions II and III.

Example-4: [bAMlAxeSe arWanIwi kaleja](/NNP) kayZati (/WQ)?(/SYM)

English gloss: How many economics colleges are in Bangladesh?

Therefore, in the Example-4 [bAMlAxeSe arWanIwi kaleja ] is the question head-word.

Now, if we consider the example "ke gOdZa prawiRTA karena?" then the syntactic features will be $[\{\mathrm{WQ}, 1\},\{\mathrm{NNP}, 1\},\{\mathrm{NN}, 1\},\{\mathrm{VM}, 1\}$, \{head-

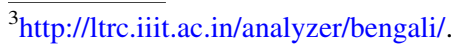


word, $\mathrm{gOdZa}\}]$. Here a feature is represented as $\{\langle$ POS, frequency $>\}$.

\subsection{Semantic features}

Semantic features $\left(f_{M}\right)$ are extracted based on the semantics of the words in a question. In this study, related words and NEs are used as $f_{M}$.

- Related word: A Bengali synonym dictionary is used to retrieve the related words. Three lists of related words were manually prepared by analysing the training data.

date: \{ janmaxina, xina, xaSaka, GantA, sapwAha, $m A s a$, baCara, ...,etc. $\}$;

food: \{ KAbAra, mACa, KAxya, mAKana, Pala,Alu, miRti, sbAxa, ..., etc.\};

human authority: \{ narapawi, rAjA, praXAnamanwrI, bicArapawi, mahAparicAlaka, ceyZAramyAna, jenArela, sulawAna, samrAta, mahAXyakRa, ..., etc.\};

If a question word belongs to any of the three lists (namely date, food, human activity), then its category name is added to the feature vector. For instance, the question "ke gedZera sbAXIna narapawi Cilena?" (gloss: who was the independent ruler of Goura?) contains the word narapawi which belongs to the human authority list. For this example question (table 2) the semantic feature is added to the feature vector as [\{human-authority, 1$\}]$.

- NEs: We used NEs as a semantic feature, which was recommended also in other works $[15,55]$ on other languages. To identify the Bengali NEs in the question text, a Margin Infused Relaxed Algorithm (MIRA)based Named Entity Recognizer (NER) [57] is used for the present study. For the Example-5 question, the NE semantic feature is added to the feature vector as [Location, 1].

Example-5: ke gOdZa[Location] prawiRTA karena? English gloss: Who established Goura?

\section{Experiments and results}

Many supervised learning approaches [13, 55, 58] have been proposed for QC over the years, but these approaches primarily differ in the classifier they use and the features they train their classifier(s) on [8]. We assume that a Bengali question is unambiguous, i.e., a question belongs to only one class. Therefore, we considered multinomial classification that assigns the most likely class from the set of classes to a question. Recent studies [12-14] also considered one label per question.

We used state-of-the-art classifier combination approaches: ensemble, stacking and voting. We have used two contemporary methods for creating accurate ensembles, namely, bagging and boosting. We employed the Rapid Miner tool for all the experiments reported here. Each of the three classifier combination approaches was tested with NB, k-NB, RI and DT classifiers.

Classification accuracy is used to evaluate the results of our experiments. Accuracy is the widely used evaluation metric to determine the class discrimination ability of classifiers, and is calculated using the following equation:

$$
\text { accuracy }=\frac{\text { number of correctly classified samples }}{\text { total number of tested samples }} \text {. }
$$

\subsection{Corpus annotation and statistics}

We carried out our experiments on the dataset described in [7]. The questions in this dataset are acquired from different domains, e.g., education, geography, history, science, etc.

Table 2. Bengali question examples.

\begin{tabular}{|c|c|}
\hline Class & Example \\
\hline Person (PER) & ke gOdZa prawiRTA karena? (gloss: Who established Goura?) \\
\hline Organization (ORG) & $\begin{array}{l}\text { sinXu saByawAra safgeV koVna saByawAra mila KuzjeV pAoVyZA yAyZa? } \\
\text { (gloss: Which civilization has resemblance with the Indus Valley Civilization?) }\end{array}$ \\
\hline Location (LOC) & gOdZa koWAyZa abashiwa? (gloss: Where is Goura situated?) \\
\hline Temporal (TEMP) & $\begin{array}{c}\text { BAikiM-2 kawa baCara karmakRama Cila? (gloss:For how many years Vaikin-2 } \\
\text { was working?) }\end{array}$ \\
\hline Numerical (NUM) & $\begin{array}{c}\text { sUrya WeVkeV Sani graheVra gadZa xUrawba kawa? (gloss: What is the average } \\
\text { distance of the planet Saturn from the Sun?) }\end{array}$ \\
\hline Method (METH) & $\begin{array}{c}\text { AryasaByawA mahilArA kiBAbeV cula bAzXawa? (gloss: How do the women } \\
\text { braid hair in the Arya Civilization) }\end{array}$ \\
\hline Reason (REA) & $\begin{array}{c}\text { AryasaByawAkeV keVna bExika saByawA balA hayZa? (gloss: Why the Arya } \\
\text { Civilization is called the Vedic Civilization?) }\end{array}$ \\
\hline Definition (DEF) & beVxa ki? (gloss: What is Veda?) \\
\hline Miscellaneous (MISC) & $\begin{array}{c}\text { Arya samAjeV cArati barNa ki ki Cila? (gloss: What are the four classes in the } \\
\text { Arya Society?) }\end{array}$ \\
\hline
\end{tabular}


We hired two native language (i.e., Bengali) specialists for annotating the corpus. Another senior native language expert was hired to support the two language specialists. The annotators were instructed to consult the senior native language expert in case of any confusion. In order to minimize disagreement, two language specialists gathered to discuss the question taxonomy in detail before initiating the annotation task. We set a constraint that a question will be annotated such that it is unambiguous, i.e., only one question class will be assigned to a question. We measured the inter-annotator agreement using non-weighted kappa coefficients [59]. The kappa coefficient for the annotation task is 0.85 , which represents very high agreement. In case of disagreement, the senior language specialist took the final decision.

The class-specific distribution of questions in the corpus is given in table 3 . It can be observed from table 3 that the most frequent question class in the dataset is 'Person'. The dataset contains a total of 1,100 questions. We divided the question corpus into $7: 3$ ratio for experimentation. The experimental dataset consists of 1,100 Bengali questions, of which $70 \%$ are used for training and the rest (331 questions, $30 \%$ ) for testing the classification models.

Table 3. Corpus statistics.

\begin{tabular}{llll}
\hline Class & Train & Test & Overall \\
\hline Person & 172 & 90 & 262 \\
Organization & 74 & 30 & 104 \\
Location & 76 & 30 & 106 \\
Temporal & 81 & 35 & 116 \\
Numerical & 71 & 30 & 101 \\
Methodical & 75 & 29 & 104 \\
Reason & 73 & 26 & 99 \\
Definition & 78 & 38 & 116 \\
Miscellaneous & 69 & 23 & 92 \\
Total & 769 & 331 & 1,100 \\
\hline
\end{tabular}

\subsection{Coarse-grained classification}

The empirical study of state-of-the-art classifier combination approaches (i.e., ensemble, stacking and voting) was performed on the said dataset using four classifiers namely NB, k-NB, RI and DT. Each experiment could be thought of as a combination of three experiments since each classifier model was tested on $\left\{f_{L}\right\},\left\{f_{L}, f_{S}\right\}$ and $\left\{f_{L}, f_{S}, f_{M}\right\}$ feature sets separately. Overall, 13 experiments are performed for coarse-grained classification and the evaluation results are reported in table 4 .

5.2a Ensemble bagging: The bagging approach is applied separately to four classifiers (i.e., NB, k-NB, RI and DT) and the obtained accuracies are summarized in table 4. Initially, the size (i.e., number of iterations) of the base learner (BL) was set to 2. Subsequently, experiments were performed with gradually increasing size (size $>2$ ). The classification accuracy enhanced with increase in size. However, after a certain size, the accuracy was almost stable. At size $=2$ and feature set $\left\{f_{L}, f_{S}, f_{M}\right\}$, the NB classifier achieved $82.23 \%$ accuracy and at size $\geq 9$, it became stable with $83.25 \%$ accuracy. At size $=2$ and feature set $\left\{f_{L}, f_{S}, f_{M}\right\}$, the k-NB classifier achieved $83.87 \%$ accuracy and at size $\geq 15$, it became stable with $84.22 \%$ accuracy. At size $=2$ and feature set $\left\{f_{L}, f_{S}, f_{M}\right\}$, the RI classifier achieved $85.97 \%$ accuracy and at size $\geq 8$, it became stable with $86.90 \%$ accuracy. At size $=2$ and feature set $\left\{f_{L}, f_{S}, f_{M}\right\}$, the DT classifier achieved $88.09 \%$ accuracy and at size $\geq 7$, it became stable with $91.27 \%$ accuracy. It was observed from the experiments that with bagging, the DT classifier performs best on any feature set for any size. For the experiments with the $f_{L}$ features, the bagging sizes of NB, k-NB, RI and DT are 12, 19, 11 and 10, respectively, after which classification accuracy becomes stable. Similarly, for experiments with $\left\{f_{L}, f_{S}\right\}$ feature set, the optimal bagging sizes are 10,17, 9 and 8 for $\mathrm{NB}, \mathrm{k}-\mathrm{NB}$, RI and DT, respectively, after which the corresponding classification accuracies converge. Figure 1

Table 4. Classifier combination results for coarse-grained classification.

\begin{tabular}{|c|c|c|c|c|c|}
\hline Approach & Base learner & Model learner & $f_{L}$ & $f_{L}+f_{S}$ & $f_{L}+f_{S}+f_{M}$ \\
\hline \multirow[t]{4}{*}{ Bagging } & NB & $\mathrm{x}$ & 81.53 & 82.77 & 83.25 \\
\hline & $\mathrm{k}-\mathrm{NB}$ & $\mathrm{x}$ & 82.09 & 83.37 & 84.22 \\
\hline & RI & $\mathrm{x}$ & 83.96 & 85.61 & 86.90 \\
\hline & DT & $\mathrm{x}$ & 85.23 & 86.41 & 91.27 \\
\hline \multirow[t]{4}{*}{ Boosting } & NB & $\mathrm{x}$ & 81.74 & 82.71 & 83.51 \\
\hline & $\mathrm{k}-\mathrm{NB}$ & $\mathrm{x}$ & 83.86 & 85.63 & 86.87 \\
\hline & RI & $\mathrm{x}$ & 83.55 & 85.59 & 86.27 \\
\hline & DT & $\mathrm{x}$ & 85.21 & 86.58 & 91.13 \\
\hline \multirow[t]{4}{*}{ Stacking } & k-NB, RI, DT & NB & 81.76 & 82.79 & 83.64 \\
\hline & NB, RI, DT & $\mathrm{k}-\mathrm{NB}$ & 83.86 & 85.54 & 86.75 \\
\hline & NB, k-NB, DT & RI & 85.55 & 87.69 & 91.32 \\
\hline & NB, k-NB, RI, & DT & 85.07 & 86.73 & 89.13 \\
\hline Voting & NB, k-NB, RI, DT & $\mathrm{x}$ & 86.59 & 88.43 & 91.65 \\
\hline
\end{tabular}




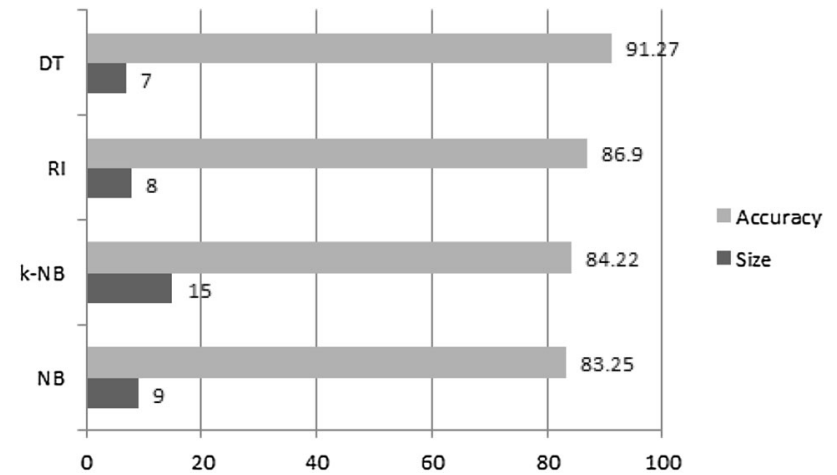

Figure 1. Size and accuracy variation in bagging with $\left\{f_{L}, f_{S}, f_{M}\right\}$.

shows the variation in size and accuracy for the best feature set.

5.2b Ensemble boosting: Like bagging, boosting (AdaBoost.M1) was also applied separately to the four base classifiers. Table 4 tabulates the accuracies obtained with the boosting approach with the four classifiers. Here, we empirically fix the iterations of boosting for the four classifiers to $12,16,10$ and 8 for the feature set $\left\{f_{L}, f_{S}, f_{M}\right\}$, since the corresponding weight of $\frac{1}{\beta_{t}}$ becomes less than 1 beyond those values. If $\frac{1}{\beta_{t}}$ is less than 1 , then the weight of the classifier model in boosting may be less than zero for that iteration. Figure 2 shows the variation in size and accuracy for the best feature set.

Similarly, for the feature sets $\left\{f_{L}, f_{S}\right\}$ and $\left\{f_{L}\right\}$ the iterations are set to $13,18,12,9$ and $14,19,14,11$, respectively, for the four classifiers. Overall the DT classifier performs the best. However, unlike in bagging, k-NB performs better than RI with boosting.

5.2c Stacking: In stacking, three out of the four classifiers are used as the base learners (BLs) and the remaining classifier is used as the model learner (ML). Therefore, four

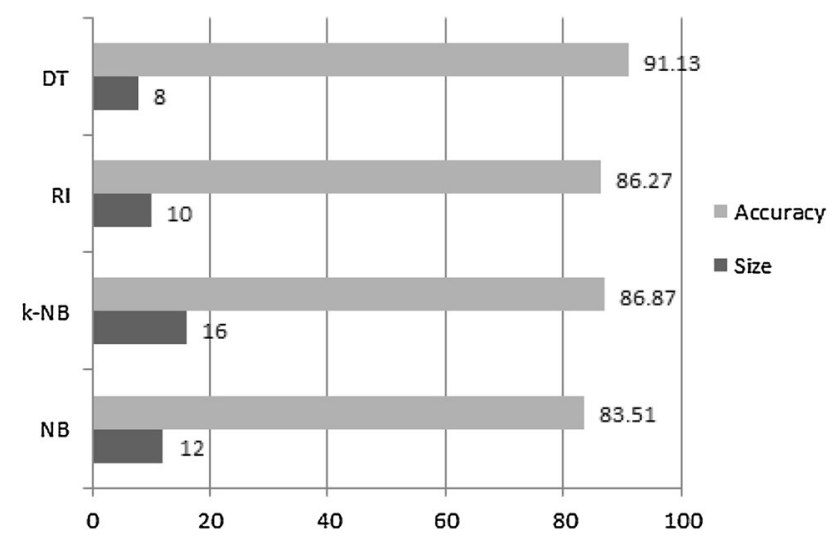

Figure 2. Size and accuracy variation in boosting with $\left\{f_{L}, f_{S}, f_{M}\right\}$. experiments were conducted separately for each of the four classifiers as the ML. The obtained accuracies are summarized in table 4.

Experimental results revealed that with RI as the ML and $\mathrm{NB}$, k-NB, DT as the BL, the classifier achieves the best classification accuracy.

5.2d Voting: In voting, four classifiers altogether were used as the BLs and majority vote was used as voting approach. The evaluation results of the voting approach are presented in table 4.

\subsection{Result analysis of coarse-grained classification}

Classifier combination is an established research known under different names in the literature: committees of learners, mixtures of experts, classifier ensembles, multiple classifier systems, etc. A number of research works $[18,19,22,24]$ established that classifier combination could produce better results than a single classifier. Generally, the key to the success of classifier combination approach is that it builds a set of diverse classifiers where each classifier is based on different subsets of the training data. Therefore, our objective is to verify the impact of the classifier combination approaches over the individual classifier approaches on Bengali QC task.

The automated Bengali QC system by [7] is based on four classifiers, namely NB, k-NB, RI and DT, which are used separately.

The experimental results obtained by [7] are shown in table 5. In that work, NB was used as the baseline and the DT classifier achieved the highest accuracy of $87.63 \%$ (see table 5). A comparison of the results in tables 4 and 5 reveals that each classifier combination model performs better than the single-classifier models in terms of classification accuracy. The prime reason is that classifier combination approaches reduce model bias and variance more effectively than individual classifiers.

In comparison with the earlier experiments reported in [7], with the bagging approach, classification accuracy of each classifier increases notably with bagging. The classification accuracy on the $\left\{f_{L}\right\},\left\{f_{L}, f_{S}\right\}$ and $\left\{f_{L}, f_{S}, f_{M}\right\}$ feature sets increases, respectively, by $1.04 \%, 0.72 \%$ and $3.64 \%$ for best performing DT classifier. Similarly, with the boosting approach, the classification accuracy for the best

Table 5. Experimental results of [7].

\begin{tabular}{lrcc}
\hline Classifier & $f_{L}$ & $f_{L}+f_{S}$ & $f_{L}+f_{S}+f_{M}$ \\
\hline NB & 80.65 & 81.34 & 81.89 \\
k-NB & 81.09 & 82.37 & 83.21 \\
RI & 83.31 & 84.23 & 85.57 \\
DT & 84.19 & 85.69 & 87.63 \\
\hline
\end{tabular}


performing DT classifiers notably increases, respectively, by $1.02 \%, 0.89 \%$ and $3.50 \%$ on $\left\{f_{L}\right\},\left\{f_{L}, f_{S}\right\}$ and $\left\{f_{L}, f_{S}, f_{M}\right\}$ feature sets. The stacking approach increases the accuracy on the $\left\{f_{L}, f_{S}\right\}$ feature set than the bagging and boosting approaches. This approach increases the classification accuracy by $1.36 \%, 2.74 \%$ and $0.69 \%$ on the $\left\{f_{L}\right\},\left\{f_{L}, f_{S}\right\}$ and $\left\{f_{L}, f_{S}, f_{M}\right\}$ feature sets, respectively. The voting approach not only increases the classification accuracy but also provides the maximum accuracy for all the feature sets than the other combined approaches. The voting approach increases the classification accuracy on the $\left\{f_{L}\right\},\left\{f_{L}, f_{S}\right\}$ and $\left\{f_{L}, f_{S}, f_{M}\right\}$ feature sets by $2.40 \%, 2.40 \%$ and $4.02 \%$, respectively. Therefore, overall, the voting approach with majority voting performed the best among the four classifier combination approaches.

Bagging approach helps avoid over-fitting by reducing variance [18]. However, after certain iterations, it cannot reduce variance. Hence, after certain iterations, it does not improve the performance of the model. Therefore, we observed that after size (i.e., number of iterations), it was unable to enhance the accuracy.

On the other hand, boosting approach enhances the performance of the model by primarily reducing the bias [60]. However, after certain iterations (size) it cannot be improved because the corresponding weight of $\frac{1}{\beta_{t}}$ becomes less than 1. If $\frac{1}{\beta_{t}}$ is less than 1 , then the weight of the classifier model in boosting may be less than zero for that iteration. Therefore, we were not able to improve the accuracy after specific boosting size.

In stacking, the ML is trained on the outputs of the BLs that are trained based on a complete training set [21]. Our experiment reveals that RI as ML and NB, k-NB, DT as the BLs outperform the other models.

In the context of Bengali QC task, we conclude from the experimental results that although classifier combination approach outperforms the individual classifier approach, the impact of different classifier combination approaches is almost same for the Bengali course classes because we obtained almost similar accuracy for different classifier combination approaches, namely, ensemble, stacking and voting.

\subsection{Fine-grained classification}

Initially, we applied NB, k-NB, RI and DT classifiers separately. Each classifier was trained with $\left\{f_{L}\right\},\left\{f_{L}, f_{S}\right\}$ and $\left\{f_{L}, f_{S}, f_{M}\right\}$ feature sets. The performance of the classifiers increases gradually with incorporation of syntactic and semantic features (i.e., $\left\{f_{L}\right\} \rightarrow\left\{f_{L}, f_{S}\right\} \rightarrow\left\{f_{L}, f_{S}, f_{M}\right\}$ ). The NB classifiers achieved around $77 \%$ of accuracy while the k-NB and RI classifiers achieved around $80 \%$ of accuracy for the fine-grained question classes. Only the DT classifier obtained more than $80 \%$ accuracy for all the question classes. The detailed evaluation results of the fine-grained QC task using individual classifier are given in table 6. The
Table 6. Fine-grained classification using individual classifiers.

\begin{tabular}{|c|c|c|c|c|}
\hline Classifier & Class & $f_{L}$ & $f_{L}+f_{S}$ & $f_{L}+f_{S}+f_{M}$ \\
\hline \multirow[t]{9}{*}{ NB } & $F_{P E R}$ & 74.07 & 75.54 & 77.07 \\
\hline & $F_{O R G}$ & 75.33 & 76.55 & 77.70 \\
\hline & $F_{L O C}$ & 76.15 & 77.02 & 77.87 \\
\hline & $F_{T E M}$ & 75.74 & 77.16 & 77.97 \\
\hline & $F_{N U M}$ & 74.61 & 75.45 & 76.55 \\
\hline & $F_{M E T H}$ & 76.35 & 77.42 & 78.50 \\
\hline & $F_{R E A}$ & 76.19 & 77.20 & 78.02 \\
\hline & $F_{D E F}$ & 76.30 & 77.45 & 78.56 \\
\hline & $F_{M I S C}$ & 75.80 & 76.95 & 77.40 \\
\hline \multirow[t]{9}{*}{$\mathrm{k}-\mathrm{NB}$} & $F_{P E R}$ & 75.72 & 77.33 & 78.41 \\
\hline & $F_{O R G}$ & 76.76 & 77.97 & 79.28 \\
\hline & $F_{L O C}$ & 77.52 & 78.55 & 79.40 \\
\hline & $F_{T E M}$ & 77.22 & 78.73 & 79.57 \\
\hline & $F_{N U M}$ & 76.09 & 76.94 & 78.05 \\
\hline & $F_{\text {METH }}$ & 77.92 & 79.14 & 80.24 \\
\hline & $F_{R E A}$ & 77.82 & 79.36 & 80.33 \\
\hline & $F_{D E F}$ & 77.99 & 79.40 & 80.43 \\
\hline & $F_{M I S C}$ & 77.37 & 78.74 & 79.60 \\
\hline \multirow[t]{9}{*}{ RI } & $F_{P E R}$ & 77.96 & 79.04 & 80.12 \\
\hline & $F_{O R G}$ & 78.29 & 79.56 & 80.75 \\
\hline & $F_{L O C}$ & 77.67 & 78.36 & 79.18 \\
\hline & $F_{T E M}$ & 79.17 & 80.76 & 81.73 \\
\hline & $F_{N U M}$ & 78.04 & 79.03 & 80.42 \\
\hline & $F_{M E T H}$ & 79.87 & 81.00 & 82.12 \\
\hline & $F_{R E A}$ & 79.62 & 80.93 & 82.06 \\
\hline & $F_{D E F}$ & 78.98 & 80.28 & 81.28 \\
\hline & $F_{M I S C}$ & 78.59 & 79.91 & 80.90 \\
\hline \multirow[t]{9}{*}{ DT } & $F_{P E R}$ & 80.37 & 82.06 & 83.61 \\
\hline & $F_{O R G}$ & 78.78 & 80.26 & 81.68 \\
\hline & $F_{L O C}$ & 78.51 & 79.63 & 80.94 \\
\hline & $F_{T E M}$ & 80.58 & 82.03 & 83.50 \\
\hline & $F_{N U M}$ & 79.00 & 80.50 & 81.85 \\
\hline & $F_{M E T H}$ & 80.62 & 82.55 & 84.47 \\
\hline & $F_{R E A}$ & 80.51 & 82.49 & 84.42 \\
\hline & $F_{D E F}$ & 79.89 & 81.07 & 82.49 \\
\hline & $F_{M I S C}$ & 79.74 & 81.72 & 84.07 \\
\hline
\end{tabular}

subsequent sections describe the experiments with classifier combination approaches.

5.4.a Ensemble bagging: In this approach, we use four classifiers as BLs individually: NB, k-NB, RI and DT. Initially, the BLs are trained using the lexical features $\left(f_{L}\right)$. Then semantic and syntactic features are added gradually for classification model generation. Therefore, three classification models were generated for each BL. Thus, altogether 12 models were prepared for bagging. Like coarsegrained classification, initially the size (number of iterations) of the BL was set to 2. Subsequently, experiments were performed with gradually increasing sizes $($ size $>2$ ). The classification accuracy increased with higher values of size. However, after certain iterations the accuracy was almost stable. For the fine-grained classes of PER coarse class (i.e., $F_{P E R}$ ), with $\left\{f_{L}, f_{S}, f_{M}\right\}$ ) feature set at size $=2$, the NB classifier achieved $81.98 \%$ classification accuracy 
and at size $\geq 9$, it became stable with $82.87 \%$ accuracy. Similarly, with $\left\{f_{L}, f_{S}, f_{M}\right\}$ feature set the k-NB, RI and DT classifiers achieved stable accuracies at size equal to 13,8 and 7, respectively. For the lexical feature set, the bagging sizes of NB, k-NB, RI and DT were 13, 20, 12 and 11, respectively, after which the classification accuracy became stable. For the combined lexical and syntactic features, the recorded bagging sizes of NB, k-NB, RI and DT were 11, 18,10 and 9 , respectively. Figure 3 depicts the iteration size for the bagging approach.

5.4b Ensemble boosting: Like the ensemble bagging approach, we applied boosting (i.e., AdaBoost.M1) separately to the four classifiers. Experimental results confirm that performances of the four base classifiers improve slightly using AdaBoost.M1. Table 7 presents the results of the boosting experiments and shows that altogether, DT outperforms the other classifiers in the ensemble approach, i.e., bagging and boosting.

In the boosting approach, the number of iterations depends on $\frac{1}{\beta_{t}}$. When the value of $\frac{1}{\beta_{t}}$ becomes less than 1 , then for that iteration the weight of the boosting classification may be less than zero. Hence, we empirically fixed the iterations of AdaBoost.M1 for the four classifiers (i.e., NB, k-NB, RI and DT) to $13,17,11$ and 9, respectively, for the feature set $\left\{f_{L}, f_{S}, f_{M}\right\}$ since the weight of $\frac{1}{\beta_{t}}$ becomes less than 1 after those values. Similarly, for feature set $\left\{f_{L}, f_{S}\right\}$ and $\left\{f_{L}\right\}$, the iterations were $14,19,13,10$ and $15,20,15,12$, respectively, for the four base classifiers. Figure 4 depicts the iteration sizes of the four classifiers in the boosting approach.

5.4c Stacking: As discussed in section 5.2c, in stacking, one classifier plays the role of ML while the remaining classifiers act as BLs. Therefore, with four classifiers, four experiments were conducted separately. The obtained accuracies are reported in table 8. From the experimental results it was observed that the model trained with DT as the ML and NB, k-NB, RI as the BLs achieved the best classification accuracy.

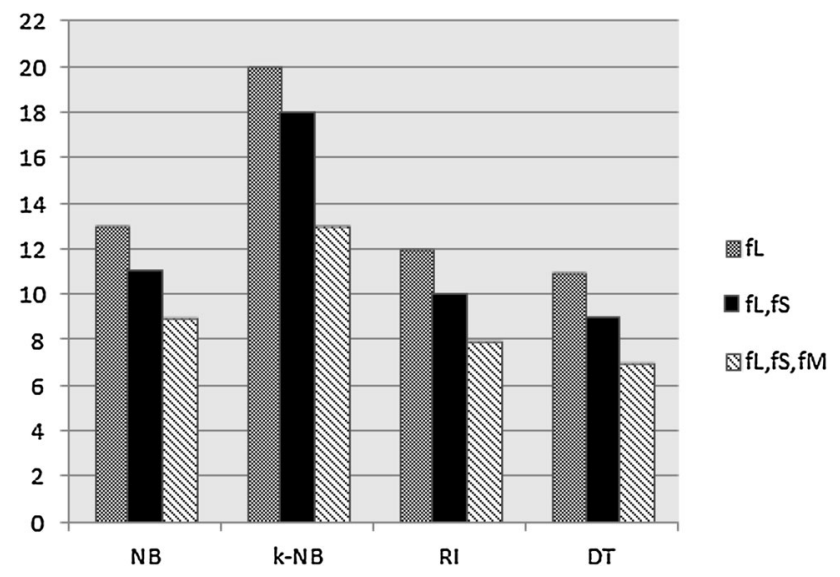

Figure 3. Size variation in bagging.
Table 7. Ensemble results of fine-grained classification.

\begin{tabular}{|c|c|c|c|c|c|c|c|}
\hline & & \multicolumn{3}{|c|}{ Bagging } & \multicolumn{3}{|c|}{ Boosting } \\
\hline & & $f_{L}$ & $f_{L}+f_{S}$ & $f_{L}+f_{S}+f_{M}$ & $f_{L}$ & $f_{L}+f_{S}$ & $f_{L}+f_{S}+f_{M}$ \\
\hline \multirow[t]{9}{*}{ NB } & $F_{P E R}$ & 79.65 & 81.23 & 82.87 & 79.89 & 81.41 & 82.95 \\
\hline & $F_{O R G}$ & 81.01 & 82.32 & 83.55 & 81.65 & 82.73 & 83.98 \\
\hline & $F_{L O C}$ & 81.89 & 82.82 & 83.73 & 82.28 & 83.85 & 85.04 \\
\hline & $F_{T E M}$ & 81.45 & 82.97 & 83.84 & 81.89 & 83.01 & 83.97 \\
\hline & $F_{N U M}$ & 80.23 & 81.13 & 82.31 & 81.02 & 81.92 & 83.03 \\
\hline & $F_{\text {METH }}$ & 82.10 & 83.25 & 84.41 & 82.25 & 83.37 & 84.53 \\
\hline & $F_{R E A}$ & 81.93 & 83.02 & 84.17 & 82.06 & 83.11 & 84.23 \\
\hline & $F_{D E F}$ & 82.05 & 83.29 & 84.47 & 82.09 & 83.32 & 84.56 \\
\hline & $F_{M I S C}$ & 81.51 & 82.75 & 83.23 & 81.62 & 82.79 & 83.75 \\
\hline \multirow[t]{9}{*}{ k-NB } & $F_{P E R}$ & 80.13 & 81.83 & 82.97 & 80.17 & 81.91 & 83.02 \\
\hline & $F_{O R G}$ & 81.23 & 82.51 & 83.89 & 81.29 & 82.63 & 83.91 \\
\hline & $F_{L O C}$ & 82.03 & 83.12 & 84.02 & 82.10 & 83.17 & 84.09 \\
\hline & $F_{T E M}$ & 81.71 & 83.31 & 84.20 & 81.79 & 83.39 & 84.28 \\
\hline & $F_{N U M}$ & 80.52 & 81.42 & 82.59 & 80.63 & 81.58 & 82.69 \\
\hline & $F_{\text {METH }}$ & 82.45 & 83.75 & 84.91 & 82.48 & 83.79 & 84.98 \\
\hline & $F_{R E A}$ & 82.35 & 83.98 & 85.01 & 82.41 & 84.02 & 85.09 \\
\hline & $F_{D E F}$ & 82.53 & 84.02 & 85.11 & 82.61 & 84.12 & 85.13 \\
\hline & $F_{M I S C}$ & 81.87 & 83.32 & 84.23 & 81.91 & 83.39 & 84.28 \\
\hline \multirow[t]{9}{*}{ RI } & $F_{P E R}$ & 81.85 & 82.98 & 84.12 & 81.92 & 83.06 & 84.22 \\
\hline & $F_{O R G}$ & 82.19 & 83.53 & 84.78 & 82.25 & 83.61 & 84.85 \\
\hline & $F_{L O C}$ & 81.54 & 82.27 & 83.13 & 81.55 & 82.26 & 83.15 \\
\hline & $F_{T E M}$ & 83.12 & 84.79 & 85.81 & 83.18 & 84.85 & 85.93 \\
\hline & $F_{N U M}$ & 81.93 & 82.97 & 84.43 & 82.01 & 83.03 & 84.49 \\
\hline & $F_{\text {METH }}$ & 83.85 & 85.04 & 86.22 & 83.91 & 85.06 & 86.31 \\
\hline & $F_{R E A}$ & 83.59 & 84.97 & 86.15 & 83.68 & 85.11 & 86.33 \\
\hline & $F_{D E F}$ & 82.92 & 84.28 & 85.33 & 82.95 & 84.32 & 85.41 \\
\hline & $F_{M I S C}$ & 82.51 & 83.89 & 84.93 & 82.57 & 83.93 & 84.98 \\
\hline \multirow[t]{9}{*}{ DT } & $F_{P E R}$ & 84.79 & 86.57 & 88.21 & 84.81 & 86.63 & 88.53 \\
\hline & $F_{O R G}$ & 83.11 & 84.67 & 86.17 & 83.14 & 84.73 & 86.23 \\
\hline & $F_{L O C}$ & 82.83 & 84.01 & 85.39 & 82.87 & 84.13 & 85.52 \\
\hline & $F_{T E M}$ & 85.01 & 86.54 & 88.09 & 85.03 & 86.58 & 88.15 \\
\hline & $F_{N U M}$ & 83.34 & 84.92 & 86.35 & 83.38 & 84.97 & 86.44 \\
\hline & $F_{M E T H}$ & 85.05 & 87.09 & 89.11 & 85.09 & 87.14 & 89.12 \\
\hline & $F_{R E A}$ & 84.93 & 87.02 & 89.06 & 84.96 & 87.11 & 89.09 \\
\hline & $F_{D E F}$ & 84.28 & 85.53 & 87.02 & 84.29 & 85.55 & 87.05 \\
\hline & $F_{M I S C}$ & 84.12 & 86.21 & 88.69 & 84.15 & 86.23 & 88.73 \\
\hline
\end{tabular}

5.4d Voting: Unlike the ensemble approach, in the voting approach, all the classifiers were applied at the same time to predict the question class. Table 9 tabulates the the accuracies obtained with this approach.

\subsection{Result analysis of fine-grained classification}

As research studies [18, 19, 22, 24] argue that classifier combination approaches provide better prediction results over individual classifier approach, our motivation is to verify the impact of the classifier combination approaches on Bengali QC task.

Initially, we carried out our experiment with individual classifier approach and applied NB, k-NB, RI and DT classifiers separately. Table 6 presents the results obtained 


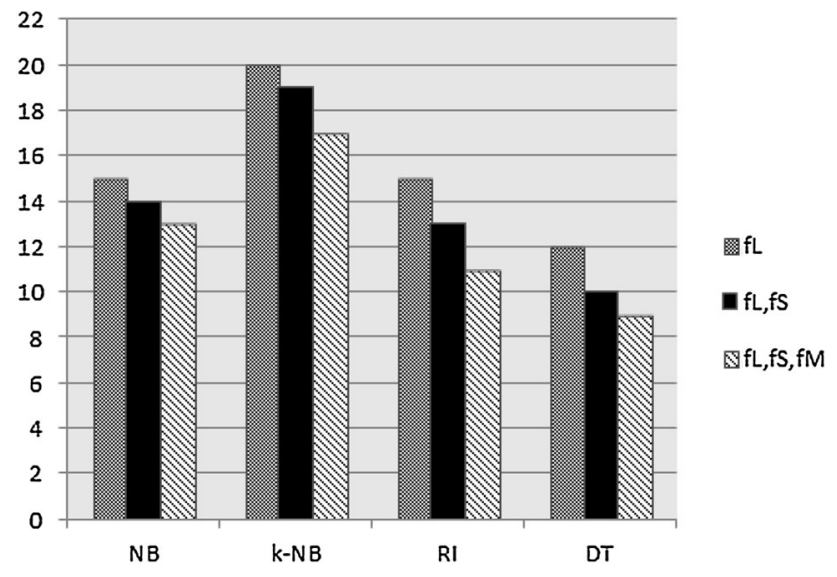

Figure 4. Size variation in boosting.

Table 8. Results of fine-grained classification with stacking.

\begin{tabular}{|c|c|c|c|c|c|}
\hline Base learner & Model learner & Class & $\mathrm{s} \quad f_{L}$ & $f_{L}+f_{S}$ & $f_{L}+f_{S}+f_{M}$ \\
\hline \multirow[t]{9}{*}{ k-NB, RI, DT } & \multirow[t]{9}{*}{ NB } & $F_{P E R}$ & 79.81 & 81.67 & 82.86 \\
\hline & & $F_{O R G}$ & 81.79 & 83.02 & 84.02 \\
\hline & & $F_{L O C}$ & 81.97 & 83.74 & 84.91 \\
\hline & & $F_{T E M}$ & 81.45 & 82.81 & 83.73 \\
\hline & & $F_{N U M}$ & 81.83 & 82.07 & 83.54 \\
\hline & & $F_{\text {METH }}$ & 82.15 & 83.13 & 84.09 \\
\hline & & $F_{R E A}$ & 82.24 & 83.36 & 84.42 \\
\hline & & $F_{D E F}$ & 81.76 & 83.05 & 84.23 \\
\hline & & $F_{M I S C}$ & 80.21 & 82.33 & 83.21 \\
\hline \multirow[t]{9}{*}{ NB, RI, DT } & \multirow[t]{9}{*}{$\mathrm{k}-\mathrm{NB}$} & $F_{P E R}$ & 79.93 & 81.79 & 83.03 \\
\hline & & $F_{O R G}$ & 81.86 & 83.16 & 84.13 \\
\hline & & $F_{L O C}$ & 82.08 & 83.82 & 85.06 \\
\hline & & $F_{T E M}$ & 81.52 & 83.01 & 83.87 \\
\hline & & $F_{N U M}$ & 81.97 & 82.18 & 83.71 \\
\hline & & $F_{\text {METH }}$ & 82.28 & 83.20 & 84.18 \\
\hline & & $F_{R E A}$ & 82.31 & 83.43 & 84.45 \\
\hline & & $F_{D E F}$ & 81.82 & 83.21 & 84.31 \\
\hline & & $F_{M I S C}$ & 80.29 & 82.42 & 83.35 \\
\hline \multirow[t]{9}{*}{ NB, k-NB, DT } & \multirow[t]{9}{*}{ RI } & $F_{P E R}$ & 80.56 & 83.06 & 84.22 \\
\hline & & $F_{O R G}$ & 82.86 & 83.98 & 85.03 \\
\hline & & $F_{L O C}$ & 80.23 & 81.49 & 82.95 \\
\hline & & $F_{T E M}$ & 83.21 & 84.78 & 85.97 \\
\hline & & $F_{N U M}$ & 82.37 & 83.42 & 84.77 \\
\hline & & $F_{\text {METH }}$ & 83.54 & 84.93 & 86.27 \\
\hline & & $F_{R E A}$ & 84.03 & 85.75 & 86.73 \\
\hline & & $F_{D E F}$ & 80.01 & 82.33 & 84.21 \\
\hline & & $F_{M I S C}$ & 82.45 & 83.86 & 84.87 \\
\hline \multirow[t]{9}{*}{$\mathrm{NB}, \mathrm{k}-\mathrm{NB}, \mathrm{RI}$} & \multirow[t]{9}{*}{ DT } & $F_{P E R}$ & 84.97 & 86.69 & 88.71 \\
\hline & & $F_{O R G}$ & 83.32 & 85.06 & 87.43 \\
\hline & & $F_{L O C}$ & 82.93 & 84.21 & 85.71 \\
\hline & & $F_{T E M}$ & 84.84 & 86.13 & 87.95 \\
\hline & & $F_{N U M}$ & 83.57 & 85.17 & 87.49 \\
\hline & & $F_{\text {METH }}$ & 84.85 & 86.91 & 88.56 \\
\hline & & $F_{R E A}$ & 84.69 & 86.78 & 88.29 \\
\hline & & $F_{D E F}$ & 84.38 & 85.65 & 87.51 \\
\hline & & $F_{M I S C}$ & 84.02 & 86.11 & 88.42 \\
\hline
\end{tabular}

Table 9. Results of fine-grained classification with voting.

\begin{tabular}{llrcc}
\hline Base learner & Class & $f_{L}$ & $f_{L}+f_{S}$ & $f_{L}+f_{S}+f_{M}$ \\
\hline NB, k-NB, RI, DT & $F_{P E R}$ & 79.81 & 81.67 & 82.86 \\
& $F_{O R G}$ & 81.79 & 83.02 & 84.02 \\
& $F_{L O C}$ & 81.97 & 83.74 & 84.91 \\
& $F_{T E M}$ & 81.45 & 82.81 & 83.73 \\
& $F_{\text {NUM }}$ & 81.83 & 82.07 & 83.54 \\
& $F_{M E T H}$ & 82.15 & 83.13 & 84.09 \\
& $F_{\text {REA }}$ & 82.24 & 83.36 & 84.42 \\
& $F_{D E F}$ & 81.76 & 83.05 & 84.23 \\
& $F_{M I S C}$ & 80.21 & 82.33 & 83.21 \\
\hline
\end{tabular}

using individual classifier approach. In fine-grained classification task, we used the identical features that were also used in coarse-grained classification. Inevitably, the obtained accuracies for fine-grained classification is less than the coarse-grained classification using the same feature sets.

Then, we applied the state-of-the-art classifier combination techniques on the lexical, syntactic and semantic feature sets. Figure 3 depicts the bagging size (i.e., number of iterations) for fine-grained classification. Breiman [18] stated that bagging approach improves the performance of a prediction model by reducing the variance. However, after certain iterations, it cannot reduce variance and the model becomes stable. Hence, after certain iterations, we were not able to improve the performance of the models. We notice that the bagging approach requires more iterations to stabilize in fine-grained classification in comparison with the coarse-grained classification. In contrast, boosting approach enhances the performance of the model by primarily reducing the bias [60]. After certain iterations, the boosting approach cannot reduce the bias because the corresponding weight of $\frac{1}{\beta_{t}}$ becomes less than 1 . If $\frac{1}{\beta_{t}}$ is less than 1 , then the weight of the classifier model in boosting may be less than zero for that iteration. Hence, in figure 4 , we can see that the boosting size is stable after certain iterations. Table 7 shows that the boosting approach achieves slightly better performance than the bagging. In stacking approach, one classifier plays the role of ML and a set of classifiers act as BLs. In the stacking approaches, the set-up with NB, k-NB, RI as BLs and DT as ML outperforms other set-up combinations. The stacking approach outperforms the voting approach with a slight margin. However, the boosting approach with the base classifier DT achieves the best. It is noticed from the fine-grained QC that all the classifier combination approaches beat the individual classifier approaches with a notable margin.

\subsection{Error analysis}

We checked the dataset and the system output to analyse the errors. We observed the following as the major sources of errors in the proposed system. 
- Questions belonging to different question classes have the same content words, which make the classifiers to get confused and wrongly classify the questions into the same class. For example, both the questions "koVna saByawAkeV bExika saByawA balA hayZa?" (gloss: which civilization is called the Vedic Civilization?), "Arya saBya-wAkeV keVna bExika saByawA balA hayZa?" (gloss: why the Arya Civilization is called the Vedic Civilization?) have the same content words: saByawAkeV, bExika, saByawA and hayZa.

- In Bengli, the dual interrogatives consist of two single interrogatives. Thus, classifiers get confused by encountering two interrogative words. Therefore, classifiers often misclassify such questions.

- The classifiers wrongly classified the Bengali questions that are long and complex. For example, 'keVna AXunika yugeVra paNdiweVrA maneV kareVna yeV, sinXu saByawA xrAbidZa jAwIra xbArA sqRti hayZeVCila? (gloss: why the modern scholars think that the Indus Valley Civilization is created by the Aryans?).

\section{Conclusions}

Although QA research in other languages (such as English) has progressed significantly, for majority of Indian languages it is at the early stage of development. In this study, we addressed the QC task for Bengali, one of the most spoken languages in the world and the second most spoken language in India. We reported experiments for coarsegrained and fine-grained QC. We employed lexical, syntactic and semantic features. We applied classifiers individually, as well as combination approaches. The automated Bengali QC system obtains up to $91.65 \%$ accuracy for coarse-grained classes and $87.79 \%$ for finegrained classes using classifier combination approaches based on four classifiers, namely NB, k-NB, RI and DT. The contributions of this work are listed as follows:

- This work successfully deploys state-of-the-art classifier combination approaches for the QC task in Bengali.

- We have empirically established the efficacy of the classifier combination approach over individual classifier approach for coarse-grained QC as well as finegrained QC.

- We have extended the single-layered (coarse-grained) taxonomy into two-layered (coarse-grained and finegrained) taxonomy by incorporating 69 fine-grained classes to the QC taxonomy.

- This work improves QC accuracy, which in turns enhances the Bengali QA system performance.

In coarse-grained $\mathrm{QC}$, overall, the voting approach with majority voting technique performs best among the four classifier combination approaches, namely bagging, boosting, stacking, and voting. However, the stacking approach produces the best results for fine-grained classification.

The only available QA dataset for Bengali contains only 1,100 questions. In future, we would like to contribute to enlarge the dataset. One of the future directions of this study is employing the state-of-the-art neural network techniques. Also, we would like to apply the approaches used in this study to other less investigated languages.

\section{Acknowledgements}

Somnath Banerjee and Sudip Kumar Naskar are supported by Digital India Corporation (formerly Media Lab Asia), MeitY, Government of India, under the Visvesvaraya Ph.D. Scheme for Electronics and IT. The work of Paolo Rosso was partially funded by the Spanish MICINN under the research project PGC2018-096212-B-C31.

\section{References}

[1] Jurafsky D and Martin J H 2014 Speech and language processing. Pearson, London

[2] Martin J H and Jurafsky D 2000 Speech and language processing, international edition 710

[3] Voorhees E M 2002 Overview of the TREC 2001 question answering track. NIST Special Publication, pp. 42-51

[4] Hovy E, Gerber L, Hermjakob U, Lin C Y and Ravichandran D 2001 Toward semantics-based answer pinpointing. In: Proceedings of Human Language Technology Research, ACL, pp. 1-7

[5] Ittycheriah A, Franz M, Zhu W J, Ratnaparkhi A and Mammone R J 2000 IBM's statistical question answering system. In: Proceedings of TREC

[6] Moldovan D, Paşca M, Harabagiu S and Surdeanu M 2003 Performance issues and error analysis in an open-domain question answering system. ACM Trans. Inf. Syst. 21(2): 133-154

[7] Banerjee S and Bandyopadhyay S 2012 Bengali question classification: towards developing QA system. In: Proceedings of the 3rd Workshop on South and Sotheast Asian Language Processing (SANLP), COLING, pp. 25-40

[8] Loni B 2011 A survey of state-of-the-art methods on question classification. Technical Report, Delft University of Technology

[9] Hull D A 1999 Xerox TREC-8 question answering track report. In: Proceedings of TREC

[10] Prager J, Radev D, Brown E, Coden A and Samn V 1999 The use of predictive annotation for question answering in TREC8. Inf. Retr. 1(3): 4

[11] Moschitti A, Quarteroni S, Basili R and Manandhar S 2007 Exploiting syntactic and shallow semantic kernels for question answer classification. In: Proceedings of the 45th Annual Meeting of the Association for Computational Linguistics, p. 776

[12] Zhang D and Lee W S 2003 Question classification using support vector machines. In: Proceedings of Research and Development in Informaion Retrieval, ACM, pp. 26-32 
[13] Huang Z, Thint M and Qin Z 2008 Question classification using head words and their hypernyms. In: Proceedings of Empirical Methods in Natural Language Processing, ACL, pp. 927-936

[14] Silva J, Coheur L, Mendes A C and Wichert A 2011 From symbolic to sub-symbolic information in question classification. Artif. Intell. Rev. 35(2): 137-154

[15] Li X and Roth D 2006 Learning question classifiers: the role of semantic information. Nat. Lang. Eng. 12(03): 229-249

[16] McCallum A, Freitag D and Pereira F C N 2000 Maximum entropy markov models for information extraction and segmentation. In: Proceedings of the International Conference on Machine Learning (ICML), vol. 17, pp. 591-598

[17] Cortes C and Vapnik V 1995 Support-vector networks. Mach. Learn. 20(3): 273-297

[18] Breiman L 1996 Bagging predictors. Mach. Learn. 24(2): 123-140

[19] Clemen R T 1989 Combining forecasts: a review and annotated bibliography. Int. J. Forecast. 5(4): 559-583

[20] Perrone M P 1993 Improving regression estimation: averaging methods for variance reduction with extensions to general convex measure optimization. Ph.D. Thesis, Brown University

[21] Wolpert D H 1992 Stacked generalization. Neural Netw. 5(2): 241-259

[22] Hansen L K and Salamon P 1990 Neural network ensembles. IEEE Trans. Pattern Anal. Mach. Intell. 12: 993-1001

[23] Krogh A, Vedelsby J et al 1995 Neural network ensembles, cross validation, and active learning. Adv. Neural Inf. Process. Syst. 7: 231-238

[24] Hashem S 1997 Optimal linear combinations of neural networks. Neural Netw. 10(4): 599-614

[25] Opitz D W and Shavlik J W 1996 Actively searching for an effective neural network ensemble. Connect. Sci. 8(3-4): 337-354

[26] Opitz D W and Shavlik J W 1996 Generating accurate and diverse members of a neural-network ensemble. In: Advances in neural information processing systems, pp. 535-541

[27] Xin L, Huang X J and Wu L 2006 Question classification by ensemble learning. Int. J. Comput. Sci. Netw. Secur. 6(3): 147

[28] Schapire R E 1990 The strength of weak learnability. Mach. Learn. 5(2): 197-227

[29] Brill E 1995 Transformation-based error-driven learning and natural language processing: a case study in part-of-speech tagging. Comput. Linguist. 21(4): 543-565

[30] Jia K, Chen K, Fan X and Zhang Y 2007 Chinese question classification based on ensemble learning. In: Proceedings of ACIS International Conference on Software Engineering, Artificial Intelligence, Networking, and Parallel/Distributed Computing, SNPD 2007. IEEE, vol. 3, pp. 342-347

[31] Su L, Liao H, Yu Z and Zhao Q 2009 Ensemble learning for question classification. In: Proceedings of Intelligent Computing and Intelligent Systems, ICIS. IEEE, pp. 501-505

[32] Ferrucci D, Brown E, Chu-Carroll J, Fan J et al 2010 Building Watson: an overview of the DeepQA project. $A I$ Mag. 31(3): 59-79

[33] Pérez-Coutiño M A, Montes-y-Gómez M, López-López A and Villaseñor-Pineda L 2005 Experiments for tuning the values of lexical features in question answering for Spanish. In: CLEF Working Notes
[34] Neumann G and Sacaleanu B 2003 A cross-language question/answering system for German and English. In: Proceedings of the Workshop of the Cross-Language Evaluation Forum for European Languages, pp. 559-571

[35] Blunsom P, Kocik K and Curran J R 2006 Question classification with log-linear models. In: Proceedings of the 29th annual international ACM SIGIR conference on Research and development in information retrieval. ACM, pp. 615-616

[36] Rosso P, Benajiba Y and Lyhyaoui A 2006 In: Proceedings of the 4th Conference on Scientific Research Outlook and Technology Development in the Arab World, pp. 11-14

[37] Abouenour L, Bouzoubaa K and Rosso P 2012 IDRAAQ: new Arabic question answering system based on query expansion and passage retrieval. In: Proceedings of CELCT

[38] Sakai T, Saito Y, Ichimura Y, Koyama M, Kokubu T and Manabe T 2004 ASKMi: a Japanese question answering system based on semantic role analysis. In: Proceedings of Coupling Approaches, Coupling Media and Coupling Languages for Information Retrieval, pp. 215-231

[39] Isozaki H, Sudoh K and Tsukada H 2005 NTT's JapaneseEnglish cross-language question answering system. In: Proceedings of NTCIR

[40] Yongkui Z, Zheqian Z, Lijun B and Xinqing C 2003 Internetbased Chinese question-answering system. Comput. Eng. 15: 34

[41] Sun A, Jiang M, He Y, Chen L and Yuan B 2008 Chinese question answering based on syntax analysis and answer classification. Acta Electron. Sin. 36(5): 833-839

[42] Sahu S, Vasnik N and Roy D 2012 Prashnottar: a Hindi question answering system. Int. J. Comput. Sci. Inf. Technol. 4(2): 149

[43] Nanda G, Dua M and Singla K 2016 A Hindi question answering system using machine learning approach. In: Proceedings of Computational Techniques in Information and Communication Technologies (ICCTICT). IEEE, pp. 311-314

[44] Sekine S and Grishman R 2003 Hindi-English cross-lingual question-answering system. ACM Trans. Asian Lang. Inf. Process. 2(3): 181-192

[45] Shukla P, Mukherjee A and Raina A 2004 Towards a language independent encoding of documents. In: Proceedings of NLUCS 2004, p. 116

[46] Ray S K, Ahmad A and Shaalan K 2018 A review of the state of the art in Hindi question answering systems. In: Proceedings of Intelligent Natural Language Processing: Trends and Applications, pp. 265-292

[47] Kumar P, Kashyap S, Mittal A and Gupta S 2003 A query answering system for e-learning Hindi documents. South Asian Lang. Rev. 13(1-2): 69-81

[48] Reddy R, Reddy N and Bandyopadhyay S 2006 Dialogue based question answering system in Telugu. In: Proceedings of the Workshop on Multilingual Question Answering, pp. 53-60

[49] Dhanjal G S, Sharma S and Sarao P K 2016 Gravity based Punjabi question answering system. Int. J. Comput. Appl. 147(3): 30-35

[50] Bindu M S and Mary I S 2012 Design and development of a named entity based question answering system for Malayalam language. Ph.D. Thesis, Cochin University of Science and Technology 
[51] Lee C W et al 2005 ASQA: academia sinica question answering system for NTCIR-5 CLQA. In: Proceedings of the NTCIR-5 Workshop, pp. 202-208

[52] Banerjee S and Bandyopadhyay S 2013 Ensemble approach for fine-grained question classification in Bengali. In: Proceedings of the 27th Pacific-Asia Conference on Language, Information, and Computation (PACLIC-27), pp. 75-84

[53] Loni B, Van Tulder G, Wiggers P, Tax D M J and Loog M 2011 Question classification by weighted combination of lexical, syntactic and semantic features. In: Proceedings of the International Conference on Text, Speech, and Dialogue, pp. $243-250$

[54] Huang Z, Thint M and Celikyilmaz A 2009 Investigation of question classifier in question answering. In: Proceedings of Empirical Methods in Natural Language Processing. ACL, vol. 2, pp. 543-550

[55] Blunsom P, Kocik K and Curran J R 2006 Question classification with log-linear models. In: Proceedings of
ACM SIGIR Conference on Research and Development in Information Retrieval. ACM, pp. 615-616

[56] Diwakar S, Goyal P and Gupta R 2010 Transliteration among indian languages using WX notation. In: Proceedings of the Conference on Natural Language Processing, EPFL-CONF-168805. Saarland University Press, pp. $147-150$

[57] Banerjee S, Naskar S K and Bandyopadhyay S Bengali named entity recognition using margin infused relaxed algorithm. In: Proceedings of the International Conference on Text, Speech, and Dialogue, pp. 125-132

[58] Li X and Roth D Learning question classifiers. In: Proceedings of the 19th International Conference on Computational Linguistics, ACL, vol. 1, pp. 1-7

[59] Cohen J 1960 A coefficient of agreement for nominal scales. Educ. Psychol. Meas. 20(1): 37-46

[60] Schapire R E 1990 The strength of weak learnability. Mach. Learn. 5(2): 197-227 